\title{
Leaking Mycotic Aneurysm of Abdominal Aorta in a 14-Year-0ld Boy with Infective Endocarditis and Severe Mitral Regurgitation-A Rare Case Report
}

\section{Harish Babu Ravulapalli1, Ramesh Srigiri², Periyasamy33, Ranjith Karthekeyan", Mahesh Vakamudi ${ }^{5}$}

${ }^{1}$ Narayana Medical College \& Hospital, Nellore, India

${ }^{2}$ Perioperative Medicine, Fakeeh University Hospital, Dubai, United Arab Emirates

${ }^{3}$ Department of Cardiothorasic Surgery, Sri Ramachandra Medical College, Porur, India

${ }^{4}$ Department of Cardiac Anaesthesiology, Sri Ramachandra Medical College, Porur, India

${ }^{5}$ Sri Ramachandra Medical College, Porur, India

Email: drharish78@gmail.com

How to cite this paper: Ravulapalli, H.B., Srigiri, R., Periyasamy, Karthekeyan, R. and Vakamudi, M. (2021) Leaking Mycotic Aneurysm of Abdominal Aorta in a 14-Year-Old Boy with Infective Endocarditis and Severe Mitral Regurgitation-A Rare Case Report. World Journal of Cardiovascular Surgery, 11, 8-15.

https://doi.org/10.4236/wjcs.2021.111002

Received: December 4, 2020

Accepted: January 17, 2021

Published: January 20, 2021

Copyright $\odot 2021$ by author(s) and Scientific Research Publishing Inc. This work is licensed under the Creative Commons Attribution International License (CC BY 4.0).

http://creativecommons.org/licenses/by/4.0/ (c) (i) Open Access

\begin{abstract}
We present a case of leaking mycotic aneurysm of abdominal aorta complicated by infective endocarditis in a young boy of 14 years age. This boy with history of rheumatic heart disease with vegetations on mitral valve and severe mitral regurgitation landed up in complications of infective endocarditis like femoral artery thrombo embolism followed by an abdominal aortic aneurysm with contained rupture. This case was successfully managed addressing two problems i.e. valve and aneurysm repair in single sitting.
\end{abstract}

\section{Keywords}

Mycotic Aneurysm, Infective Endocarditis

\section{Introduction}

Abdominal aortic aneurysms are rare in children, causes include Mycotic Aneurysm, vasculitides (e.g.: Takayasu's arteritis) connective tissue diseases (e.g.: Marfan's syndrome, Ehler-Danlos syndrome and tuberous sclerosis) and traumatic false aneurysms [1]. The overall incidence of mycotic aneurysms in patients with infective endocarditis is in between 2 to 10 percent; in 1 to 5 percent the aneurysms involve cerebral vessels [2]. The exact incidence of extra cranial vessels involvement is not known. In 1885, Osler described a case of mycotic 
aneurysm that originated from infective endocarditis [3]. With the advent of antibiotic era and aggressive management of infective endocarditis, the development of mycotic aneurysms is uncommon.

Mycotic aneurysms may resolve during antimicrobial therapy [4], however, when anatomically feasible, aneurysms that have ruptured should be repaired surgically [5]. Aneurysms that have not leaked should be monitored angiographically during antimicrobial therapy. Anticoagulant therapy should be avoided in patients with persisting mycotic aneurysms. On rare occasions, persistant stable aneurysms may rupture after completion of standard antimicrobial therapy: however, there is no accurate estimation of risk for late rupture, and recommendations for surgical intervention are arbitrary.

In native valve infective endocarditis with heart failure, the mortality rates are around 55 to 85 percent with medical treatment alone and 10 to 35 percent in operated patients [6]. Not only does heart failure increase the mortality rate if there is no surgery, but it also increases the perioperative mortality rate from 5 to 10 percent in patients without heart failure to 15 to 35 percent in patients with heart failure [7].

The objective is to highlight the serious and rare complications associated with neglected/untreated squeals of endocarditis and to share the successful management of two major complications in single setting.

\section{Case Report}

This 14 year old boy has come to our hospital with complaints of abdominal distension and vague abdominal discomfort for one day. He also gives history of breathlessness which was worsening over six months, and presented with NYHA grade III. His past history revealed that he is a known case of Rheumatic heart disease with moderate mitral regurgitation managed medically with Tab.Digoxin $0.25 \mathrm{mg}$ per oral o.d, Tab.Fruselac (Frusemide $20 \mathrm{mg}+$ Spironolactone $50 \mathrm{mg} 1$ tab per oral o.d, Tab.Enalapril $2.5 \mathrm{mg}$ per oral o.d. He also gave history of high grade continuous fever which was managed with antipyretics and antibiotics in a private clinic. This boy was previously admitted in our hospital one month before with claudicating pain in right lower limb, Doppler examination showed a thrombus in right femoral artery and subsequently he has undergone trans femoral embolectomy. He was put on oral anticoagulants with Tab.Acitrom (Nicoumalone) $1 \mathrm{mg}$ o.d. Two weeks after embolectomy he developed high grade fever with chills, blood cultures revealed the growth of Klebsiella and was treated with antibiotics according to culture sensitivity. Repeat blood cultures after one week of antibiotics showed no growth. Echocardiogram revealed dilated Left Atrium (size $38 \mathrm{~mm}$ ), vegetations over anterior Mitral leaflet $(0.9 \times 0.7 \mathrm{~cm})$, severe mitral regurgitation, mild tricuspid regurgitation with mild pulmonary artery hypertension with systolic Pulmonary artery pressure around $46 \mathrm{mmhg}$, Left ventricular ejection fraction $76 \%$, no clot, minimal pericardial effusion. In view of embolic event, severity of mitral regurgitation and vegetations over mitral valve he was advised surgery to repair mitral valve and removal of vegetations 
but his parents deferred for surgery in view of financial constrains and went home.

After one month of discharge from the hospital he came with complaints of abdominal distension and discomfort. On clinical examination the boy was poorly built and nourished weighing 25 kilograms. He was not pale, not cyanosed and no lymphadenopathy. There was grade II clubbing, JVP was raised and no pedal edema. General examination showed a swollen left knee joint which was warm and tender to touch with limitation of extension of joint. His vitals were: pulse rate-128/minute, regular, peripheral pulses and femoral pulses were not felt. B.P-90/46 mmhg in left upper limb in supine position, he was afebrile and respiratory rate was 22 /minute. Sytemic examination revealed hyperdynamic apical impulse in left 5th intercostal space lateral to nipple and grade IV pansystolic murmur over mitral area radiating to axilla. Respiratory system was normal. No focal neurological deficits. Abdomen was distended and a swelling was palpable in left iliac region which was pulsatile, tender to touch. Bowel sounds were heard. His blood investigations revealed hemoglobin of $9.1 \mathrm{grams} / \mathrm{dl}$, total white cell count of 15,330/cumm, platelet count of 2.29 lakhs/cumm, renal and liver function tests were within normal limits. Coagulation profile also normal. C-reactive protein $1.2 \mathrm{mg} / \mathrm{l}$, ASO titre $<200$ units $/ \mathrm{ml}$, Electrocardiogram (ECG) showed sinus tachycardia with left ventricular strain pattern. Contrast enhanced computed tomogram of abdomen revealed a large retroperitoneal hematoma of $16 \times 16 \times 15 \mathrm{~cm}$ size on the left side extending posteriorly (Figure 1), eroding left transverse process of Lumbar 4 vertebra (Figure 2), with extra vasation of contrast from abdominal aorta after origin of inferior mesenteric artery into the hematoma. Aortic bifurcation was not visualized, common, external and internal iliac arteries were reformed from infrarenal aorta anteriorly. Hematoma seemed to displace left kidney superiorly and laterally. Inferior vena cava is compressed by hematoma and displaced anteriorly. Hematoma appeared to sealed off with thickest wall at sides (pseudo aneurysm formation). Echocardiogram was consistent with previous findings (Figure 3). In view of patient's condition and leaking aneurysm emergency surgery was planned under general anesthesia and cardio pulmonary bypass to repair mitral valve and aneurysm of abdominal aorta. Patient was shifted to the operating theatre, an 18 gauge IV line secured in left cubital fossa, under local anesthesia left radial artery was cannulated for continuous blood pressure monitoring (IBP). Pre-induction monitoring included ECG, Pulse oximetry, Bispectral index (BIS), invasive blood pressure, Central venous pressure. A 7 french triple lumen catheter (Biosensors international PTE LTD) introduced in right internal juglar vein and pre induction hemodynamic parameters were heart rate 120/minute, IBP-88/56 mmhg, $\mathrm{SPO}_{2}-100 \%$ with 6 litres of oxygen via facemask, CVP 8 - $10 \mathrm{mmhg}$. Before induction 4 units of packed cells, 1 litre of 6\% hydroxy ethyl starch (volulyte, Fresiniuskabi, Hongkong, Zuellig) and enough quantity of crystalloids were kept ready. Cardio pulmonary bypass pump was assembled and circuit primed. After 3 minutes of pre oxygenation patient was induced with Inj.Midazolam $0.5 \mathrm{mg}$, 


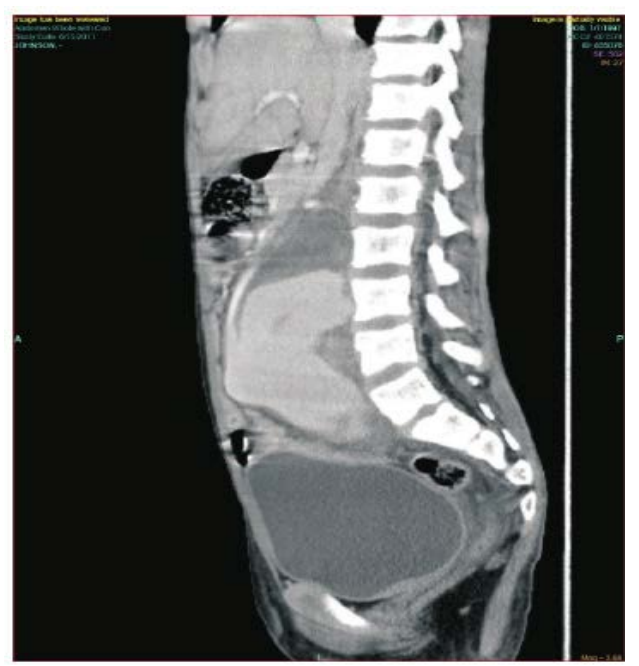

Figure 1. CT aortogram showing leaking of contrast into the haematoma.

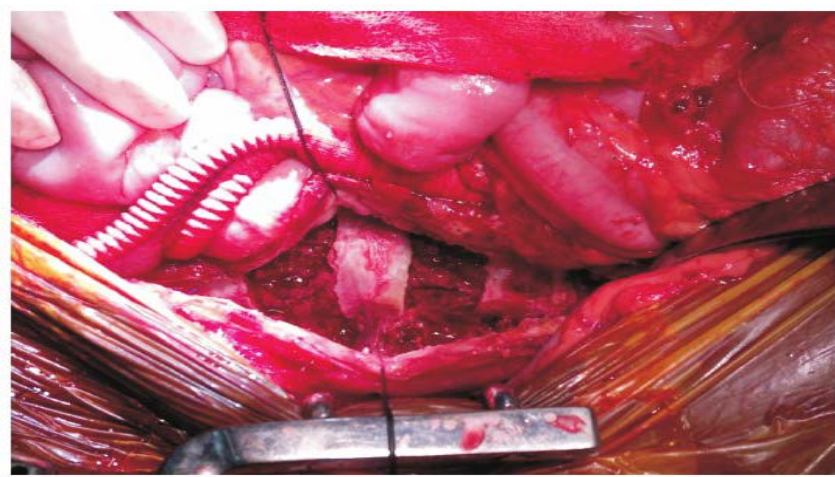

Figure 2. Erosion of L4 vertebra by haematoma.

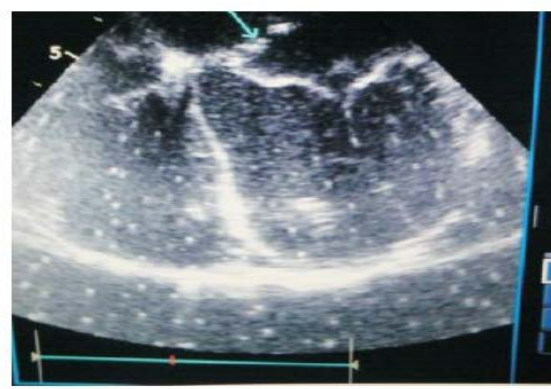

(a)

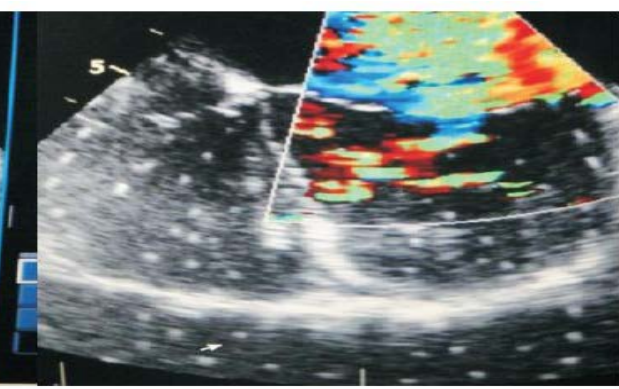

(b)

Figure 3. TEE image of midoesophageal four chamber view showing (a) Vegetation over anterior mitral leaflet, (b) severe mitral regurgitation.

Inj.Fentanyl $75 \mathrm{mcg}$, Inj.Ketamine $40 \mathrm{mg}$, titrated to hemodynamics. Inj.Vecuronium $4 \mathrm{mg}$ bolus was given to facilitate endotracheal intubation. Patient was intubated with appropriate size $(6.5 \mathrm{~mm}$ cuffed) endotracheal tube. Anesthesia was maintained with oxygen/Nitrous oxide (50/50) and sevoflorane $0.5 \%$ to $2 \%$ titrated to $1 \mathrm{MAC}$ and intermittent doses of Inj.Vecuronium and Inj.fentanyl. Post intubation patient's hemodynamic parameters were stable. 
Endtidal carbondioxide (ET $\left.\mathrm{CO}_{2}\right)$, airway pressures, urine output, nasopharyngeal temperature and arterial blood gases were monitored intra operatively along with pre induction monitors. A paediatric trans esophageal probe (9T GE vivid7, Norway) was introduced post intubation. Findings of Transesophageal echocardiogram (TEE) were consistent with preoperative Transthoracic echocardiogram.

After median sternotomy pericardium opened in the midline, after systemic Heparinization $(400 \mathrm{u} / \mathrm{kg}$ ) and adequate activated coagulation time (ACT) aortic and bicaval cannulation done, cardio pulmonary bypass initiated and patient cooled to $20^{\circ} \mathrm{C}$. Aorta cross clamped and antegrade cold blood cardioplegia given. Through trans septal approach mitral valve inspected, vegetations were removed, perforation of A3 segment of anterior mitral leaflet closed, quadrangular resection around P3 segment was done. Mitral valve checked by saline flush, the repair was unsatisfactory then the surgeon decided to put $28 \mathrm{~mm}$ carpentieredwards physio ring (Edwards life sciences, Irvine CA, U.S.A) and mitral annuloplasty was completed. Mitralvalve rechecked and repair was satisfactory. Abdomen opened through left para median incision, large aneurysmal sac found with distorted proximal aorta, distal aorta could not be visualized. To get control over aorta patient was further cooled down to $15^{\circ} \mathrm{C}$ and transient circulatory arrest was initiated for 5 minutes, proximal aorta looped aneurismal segment opened and clot evacuated, distal aortic segment was visualized and rewarming started. Aortic cross clamp was released and healthiest portion of aorta was transected just above the aneurysm. A $12 \times 6 \mathrm{~mm}$ collagen coated woven polyster Y graft (Figure 4) (Intergard, Maquet getinge group, France) anastomosed through end to end fashion, distal ends were anastomosed to external iliacs. Mouth of common iliac was closed. A side biting clamp was used during anastomosis on aorta. Hemostasis secured. Patient rewarmed to $37^{\circ} \mathrm{C}$. Heart picked up sinus rhythm. Trans esophageal echo showed mild mitral regurgitation, no vegetations and moderate left ventricular dysfunction. Patient weaned off from bypass with inotropic supports of inj.adrenaline $0.1 \mathrm{mcg} / \mathrm{kg} /$ minute, inj.dobutamine 5 $\mathrm{mcg} / \mathrm{kg} / \mathrm{minute}$ and inj.glycyryl trinitrate $2 \mathrm{mcg} / \mathrm{kg} / \mathrm{minute}$. The total amount of blood loss was 1.5 litres, urine output was 3 litres. Total of 3 units packed cells, 3 units of fresh frozen plasma, $500 \mathrm{ml}$ of ringers lactate were transfused intraoperatively. The total $\mathrm{CPB}$ time was $4 \mathrm{hrs} 22 \mathrm{mnts}$, aortic cross clamp time was 92

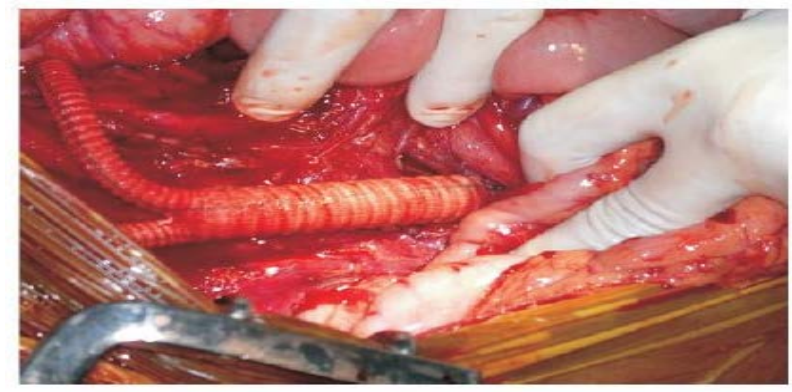

Figure 4. Collagen coated woven polyster Y graft in place. 
mnts and circulatory arrest was for 5 mnts. Patient was shifted to intensive care unit (ICU) with stable hemodynamics. Total duration of ventilation in ICU was 39 hrs 50 mnts, duration of inotropic support was for 3 days and length of ICU stay was for 5 days. Total post operative drainage was around $1850 \mathrm{ml}$. Patient was discharged from hospital on 10th post operative day and a check aortogram was done 3 months later during follow up showed good graft function echocardiogram showed mild mitral regurgitation and tricuspid regurgitation with improved left ventricular function.

\section{Discussion}

Abdominal aortic aneurysms secondary to infectious cause (mycotic aneurysms) is a rare presentation. The pathogenesis of mycotic aneurysms may include five different mechanisms [8], 1) septic embolization lodged in the vasovasorum or vessel lumen, 2) a contiguous inflammatory process outside the vessel wall that extends to the nearby artery, 3) inoculation of bacteria at the time of accidental arterial trauma, 4) Vascular manipulation or iatrogenic causes and 5) an intimal defect such as an atherosclerotic plaque, that is seeded by concurrent bacteremia.

Most of the abdominal aortic aneurysms are asymptomatic but might present as sudden rupture or as secondary complications. Our patient presented with secondary complications apart from pulsatile swelling in the lower abdomen. Less than 5 percent of patients with abdominal aortic aneurysms have evidence of arterial insufficiency to legs [9] (thrombosis of aneurysm or embolization of thrombus to leg arteries). Apart from size, signs of impending rupture like severe abdominal pain, tenderness over swelling or evidence of leak from aneurysms mandates emergency repair of aneurysm [9]. Along with thrombotic complications this patient also had contracture of leg due to psoas spasm and erosion of transverse process of L4 vertebra both due to retroperitoneal extension of hematoma. Since patient is a known case of rheumatic heart disease with heart failure (NYHA CLASS III), severe mitral regurgitation complicated by bacterial endocarditis and history of embolic events it was decided to repair mitral valve and abdominal aortic aneurysm in single setting.

The first indication for cardiac surgery in infective endocarditis is heart failure. It represents more than half of the indications. The embolism rate varies from $10 \%-50 \%$ in different series [10]. Three quarters of embolisms occur before the beginning of antibiotic treatment [11]. The embolic risk decreases greatly and regularly overtime from $15 \%$ after one week of treatment to $1 \%$ after four weeks of treatment [12]. In mitral infective endocarditis, occurrence of embolism is more frequent if vegetations are located over the anterior mitral leaflet than over the posterior mitral leaflet. In a study 10 embolic events were more frequent when the size of vegetations was more than $10 \mathrm{~mm}$ or when vegetations were very mobile.

Rapid surgery must be performed when there has been an embolic event and when large and mobile vegetations remain. When there are large and/or mobile vegetations but no embolic event, the medical community is divided regarding 
surgical intervention. The decision is clear in the case of mitral infective endocarditis when the valve repair looks possible, or when there are other arguments for surgery (heart failure, no control of infection, severe valvar and perivalvar lesions). In the present case the complication of mycotic aneurysm of abdominal aorta would have been avoided provided the patients' parents could agree for surgical intervention after the first episode of embolism to the right leg.

Severe heart failure, hemodynamic deterioration, impending rupture of aneurysm with secondary complications, careful fluid balance, control of bleeding were challenging issues in the management of present case. The patient is being followed up for three months and a check aortogram revealed good graft function and echocardiogram showed trivial to mild mitral regurgitation with improved left ventricular function.

Joo Seop Kim, Min-Kyung Kang1, A. Jin Cho [13] et al. reported a case series of mycotic aneurysms and their management and in one case the patient developed a peripherally inserted central catheter (PICC) related right sided heart valve ie Tricuspid valve endocarditis and subsequent abdominal aortic aneurysm in which case the patient deferred surgery and outcome was not known.

In view of rarity of mycotic aneurysm in this era of antibiotics we hardly found any similar case like ours in the literature.

\section{Conclusion}

Complicated infective endocarditis is rare and is associated with cardiac, neurologic, renal, musculoskeletal, and systemic complications related to infection, such as embolization, metastatic infection, and mycotic aneurysm. Infective endocarditis caused by Staphylococcus aureus is more frequently associated with complications. Because the mortality rate increases when complications develop, aggressive antibiotic therapy and surgery, combined with specific treatments for the complications, are necessary.

\section{Conflicts of Interest}

The authors declare no conflicts of interest regarding the publication of this paper.

\section{References}

[1] Millar, A.J.W., Gilbert, R.D., Brown, R.A., et al. (1996) Abdominal Aortic Aneurysms in Children. Journal of Peadiatric Surgery, 31, 1624-1628. https://doi.org/10.1016/S0022-3468(96)90034-2

[2] Karchmer, A.W. (2014) Infective Endocarditis, Braunwald's Heart Disease. A Textbook of Cardio Vascular Medicine. Saunders, Philadelphia, Pennsylvania, 1651-1652.

[3] Osler, W. (1885) The Gultonian Lectures on Malignant Endocarditis. British Medical Journal, 1, 467-470. https://doi.org/10.1136/bmj.1.1262.467

[4] Bayer, A.S., Bolger, A.F., Taubert, K.A., et al. (1998) Diagnosis and Management of Infective Endocarditis and its Complications. Circulation, 98, 29-36.

https://doi.org/10.1161/01.CIR.98.25.2936 
[5] Phoung, L.K., Link, M. and Wijdicks, E. (2002) Management Intra Cranial Infections Aneurysm: A Series of 16 Cases. Neurosurgery, 51, 1145. https://doi.org/10.1097/00006123-200211000-00008

[6] Olaison, L. and Petterson, G. (2002) Current Best Practice and Guidelines Indications for Surgical Intervention in Infective Endocarditis. Infectious Diseases Clinics of North America, 16, 453-475. https://doi.org/10.1016/S0891-5520(01)00006-X

[7] Delahaye, F., Celard, M., Roth, O., et al. (2004) Indications and Optimal Timing for Surgery in Infective Endocarditis. Heart, 90, 618-620. https://doi.org/10.1136/hrt.2003.029967

[8] Johansen, K. and Devin, J. (1983) Mycotic Aneurysms: A Reappraisal. Archives of Surgery, 118, 583-588. https://doi.org/10.1001/archsurg.1983.01390050059011

[9] Townsend, C.M., Beauchamy, R.D., Evers, B.M. and Mattox, K.L. (2001) Sabiston Textbook of Surgery: The Biological Basis of Modern Surgical Practice. Saunders Publishers, Amsterdam, 1357-1367.

[10] Di Salvo, G., Habib, G., Pergola, V., et al. (2001) Echocardiography Predicts Embolic Events in Infective Endocarditis. Journal of American College of Cardiology, 37, 1069-1076. https://doi.org/10.1016/S0735-1097(00)01206-7

[11] Heiro, M., Nikoskelainen, J., Engblom, E., et al. (2000) Neurologic Manifestations of Infective Endocarditis. A 17 Year Experience in a Teaching Hospital in Finland. Archives of Internal Medicine, 160, 2781-2787. https://doi.org/10.1001/archinte.160.18.2781

[12] Alestig, K., Hogevik, H. and Olaison, L. (2000) Infective Endocarditis: A Diagnostic and Therapeutic Challenge for the New Millennium. Scandinavian Journal of Infectious Diseases, 32, 343-356. https://doi.org/10.1080/003655400750044908

[13] Kim, J.S., Kang, M.-K., Cho, A.J., Seo, Y.B. and Kim, K.I. (2017) Complicated Infective Endocarditis: A Case Series. Journal of Medical Case Reports, 11, 128. https://doi.org/10.1186/s13256-017-1274-7 